\title{
Gradenigo Syndrome from Acute Sphenoiditis: A Case Report
}

\author{
Levente S. Deak ${ }^{1 *}$, Jaikishan Mordani ${ }^{2}$, Nanor Tchaghlasian ${ }^{1}$ and David William Saxton ${ }^{4}$ \\ ${ }^{1}$ Department of Otolaryngology, Al Zahra Private Hospital, UAE \\ ${ }^{2}$ Department of Radiology, Al Zahra Private Hospital, UAE \\ ${ }^{4}$ Chief Medical Director, Al Zahra Private Hospital, UAE \\ Submission: June 09, 2016; Published: June 22, 2016 \\ *Corresponding author: Levente S. Deak, Department of Otolaryngology, Al Zahra Private Hospital, Sheikh Zayed Road, P. 0. Box 124412, Al \\ Barsha 1, Dubai, UAE, Tel: +971 4378 6666; Ext: 6205; +971 56 3426552; Email: Levente.deak@azhd.ae
}

\begin{abstract}
Infection of paranasal sinuses may spread to the petrous bone and can present as Gradenigo syndrome. It is commonly associated with otitis media, however; due to its anatomical position, sphenoid infection should also be considered. Case Presentation: This case report reports a case of a 31 yrs old woman who presented with severe unilateral retro-ocular pain and short term facial paresthesia along with diplopia and a history of massive nasal blockage for more than 10 days. Nasal culture indicated growth of Hemophilus Influenza and a computed tomography (CT) scan confirmed acute infection of the left sphenoethmoid region and apex of the petrous bone. The mastoid cavity and the ear status was unremarkable. The patient was treated with third generation intravenous (IV) cephalosporin and high dosage corticosteroids, along with proper drainage of the sphenoid cavity by functional endoscopic sinus surgery. The case was successfully managed without any long term complication.
\end{abstract}

Keywords: Apical Petrositis; Acute Sphenoiditis; Endoscopic Sinus Surgery; Gradenigo Syndrome; Petrous Bone

Abbreviations: CT: Computed Tomography; IV: Intravenous; MRI: Magnetic Resonance Imaging MRI; HIV: Human Immunodeficiency Virus

\section{Introduction}

Anatomically, the apex of the petrous bone can be exposed by infection, benign tumors, or malignant lesions. Infection can spread to the apex from the middle ear as well as from surrounding area for example, the sphenoid sinus [1]. Apical petrositis can also present as Gradenigo's syndrome. It was first described in 1904 by Giuseppe Gradenigo and characterized by a classical clinical triad of retro-ocular pain, discharging ear and abducens nerve palsy [2]. Petrositis is considered a potentially fatal disease since it can cause meningitis, cranial nerve palsy, intracranial abscess, cavernous sinus thrombosis and hydrocephalus $[3,4]$. There has been a decrease in the number of reported cases due to early administration of antibiotics for the ear disease, but it is still a concern in immune compromised patients [4].

On the other hand the incidence of sinus-related infection and its complications have shown an increasing trend [5]. There is limited literature on the progression of acute sinusitis to the intracranial complications of Gradenigo's syndrome or apical petrositis. Infection in the petrous bone can be diagnosed by computed tomography (CT) and magnetic resonance imaging (MRI)
[6]. Traditionally, major surgical interventions was suggested for acute petrositis. However a gradual shift has been observed towards timely intervention with improved antibiotic therapy to allow conservative management of certain cases $[7,8]$. In our case report apical petrositis occurred after acute sphenoiditis and recovered without any sequelae following maximal conservative therapy and minimal invasive intervention as per as sphenoiditomy Type 2 and septoplasty.

\section{Case Presentation}

A 31 yr old woman presented with severe unilateral deep pain around the eye and short term facial paresthesia together with temporary double vision. Previously, the patient had recurrent attach of acute rhinosinusitis for which conventional treatment was given. The patient did not have any comorbid conditions such as diabetes. On examination, her vital signs were unremarkable. Significant nasal congestion with purulent secretion was seen, along with normal tympanic membranes and no tenderness was noted around the sinus and mastoid region. The patient reported severe deep dull retro-ocular pain which existed for more than 5 days. Neurological examination showed reduced sensation of the 


\section{Global Journal of Otolaryngology}

$\mathrm{V} / 2$ division of trigeminus nerve and incomplete abducens paresis. The patient did not have increased inflammatory markers. A nasal swab showed a heavy growth of Hemophilus Influenza. The patient's immunological status for human immunodeficiency virus (HIV), diabetes mellitus, and all immunoglobulin levels were normal.

The CT and MRI scan indicated an acute infection of the paranasal sinuses with full opacification of spheno-ethmoid region and maxillary sinus in addition to inflammation in the aerated apex of the petrosus bone (Figure 1A-1C). After admission to the hospital, the patient received intravenous (IV) third generation cephalosporin (ceftriaxone $2 \mathrm{gm}$ twice daily) along with IV corticosteroids (dexamethasone $16 \mathrm{mg}$ ). Twenty four hours after admission, the patient underwent endoscopic sinus surgery for drainage of sphenoid infection (septoplasty with sphenoidotomy type 2) and to maintain an open airway. The abducens paresis recovered within $12 \mathrm{~h}$ of admission and the pain level reduced significantly. Oral steroids were discontinued after 7 days and the patient was discharged from the hospital while still on IV antibiotic treatment. The patient again developed deep dull ear pain for which a repeat MRI was conducted on the 10th day. (Figures 1D-1F) illustrate post op MRI and CT scans respectively. Adequate regression of inflammation of the petrous bone is seen. The patient was restarted on oral prednisolone $40 \mathrm{mg}$, along with antibiotics (IV, once daily) for 3 weeks. After 2 weeks of treatment and an improvement in pain, the oral steroids were gradually tapered off. The MRI scan done after 1 year shows total resolution of inflammation of the sphenoid sinus and petrous apex (Figure 1C).

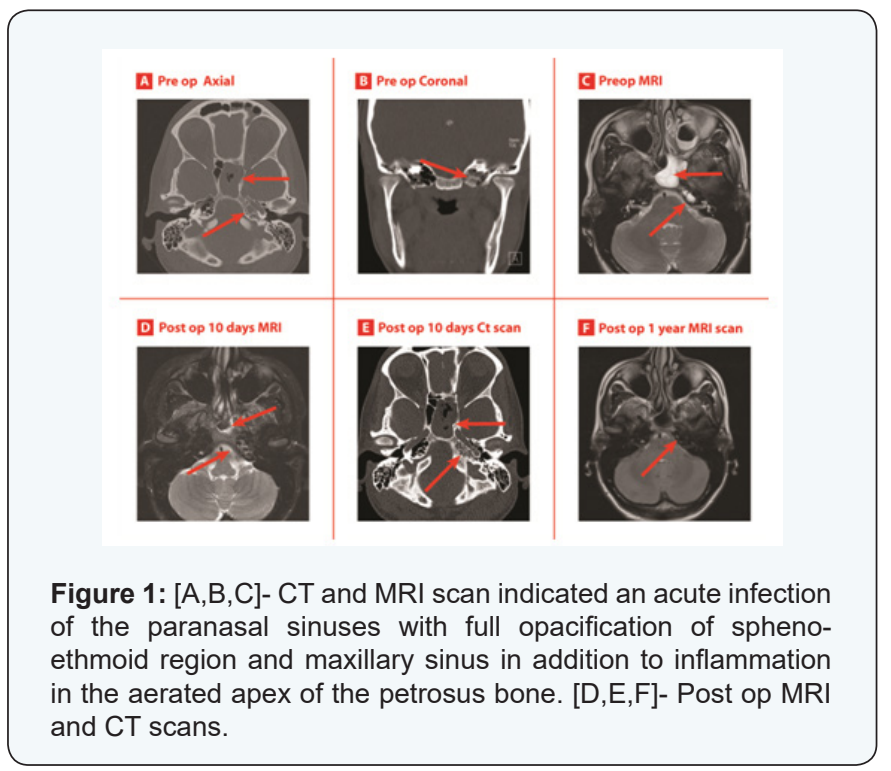

\section{Discussion}

Apical petrositis is associated with severe and life-threatening complications such as meningitis, lateral sinus thrombosis, and brain abscess [4]. Infection of the petrous bone is most commonly linked to ear infections [8-10], however, it's anatomical associa- tion with the sphenoid sinus cannot be overlooked $[1,11,12]$. In the present case, the infection originated from the sphenoid sinus and probably spread by pneumatized air cell tract or through vascular channels to the petrous apex of temporal bone, leading to apical petrositis $[12,13]$. The standard treatment regime consists of radical surgical intervention in combination with antibiotic therapy [9]. However, satisfactory improvement without surgical interventions has also been described. Use of antibiotics has changed the incidence of disease leading to the more efficient management of petrous apex infection [6,8]. Yozu A et al. [14] reported a case of a 6 year old girl with Gradenigo syndrome having widespread inflammation involving the paranasal sinuses and middle ear. The patient was managed conservatively with antibiotics and surgical treatment was avoided.

Hananya S and Horowitz Y reported a case of successful prolonged conservative management of Gradenigo syndrome in a 5 year old boy who had petrositis and cavernous sinus thrombosis as a combined complication of acute otitis media [15]. Scardapane A et al. [16] reported successful conservative treatment in a 4-year-old child who had Gradenigo's syndrome with lateral venous sinus thrombosis. Recent literature has reported satisfactory outcomes in patients with Gradenigo's syndrome after intervention with broad spectrum antibiotics and less aggressive surgeries [17].

The use of antibiotics along with surgical intervention is recommended for the management of non-responsive cases of chronic infections or with severe osteomyelitis or abscess formation [10]. In our case, after initial antibiotic and steroid intervention, the patient underwent minimal invasive endoscopic sinus surgery to drain the sphenoid sinus. The use of a low risk combined treatment model, as compared to traditional high risk extended surgical intervention resulted in a dramatic improvement of the post treatment quality of life outcome. Usually, abducens nerve palsy is the last feature to resolve, and can persist for several months. In our case, the double vision was just a temporary feature with total recovery after steroid administration. This rapid recovery has been reported previously [18]. The recommended duration for antibiotic use in the case of acute petrositis is still not clear. In the literature, both short and long term administration are practiced [18]. In our case, the quick and rapid response to conservative treatment was confirmed by MRI on $10^{\text {th }}$ day and the lack of symptoms led us to use the antibiotic treatment for three weeks.

\section{Conclusion}

This case report demonstrates a rare case of Gradenigo's syndrome beyond the classical triad, where acute sinusitis led to apical petrositis. It was managed successfully by providing wide drainage of sphenoid cavity along with maximal conservative treatment in the form of antibiotics and steroids.

\section{Informed Consent}

A written informed consent from the patient was obtained. A copy of the written consent will be available on request for review. 


\section{References}

1. Pedram Kordrostami, Amit Parmar (2014) Isolated Sphenoiditis presenting with Abducens nerve palsy. Annals of Otolaryngology and Rhinology 1(3): 1014.

2. Gradenigo G (1907) Über die Paralyse des Nervus abducens bei Otitis. Archiv für Ohrenheilkunde 74(1): 149-187.

3. Price T, Fayad G (2002) Abducens nerve palsy as the sole presenting symptom of petrous apiciti. J Laryngol Otol 116(9): 726-729.

4. Motamed M, Kalan A (2000) Gradenigo's syndrome. Postgrad Med J 76(899): 559-560.

5. Fokkens WJ, Lund VJ, Mullol J, Bachert C, Alobid I, et al. (2012) EPOS 2012: European position paper on rhinosinusitis and nasal polyps 2012. A summary for otorhinolaryngologists. Rhinology 50(1): 1-12.

6. Murakami T, Tsubaki J, Tahara Y, Nagashima T (1996) Gradenigo's syndrome: CT and MRI findings. Pediatr Radiol 26(9): 684-685.

7. Lutter SA, Kerschner JE, Chusid MJ (2005) Gradenigo syndrome: a rare but serious complication of otitis media. Pediatr Emerg Care 21(6): 384-386.

8. Burston BJ, Pretorius PM, Ramsden JD (2005) Gradenigo's syndrome: successful conservative treatment in adult and paediatric patients. J Laryngol Otol 119(4): 325-329.

9. Tutuncuogle S, Uran N, Kavas I, Ozsur T (1993) Gradenigo's syndrome; a case report. Pediatr Radiol 23: 556.

10. Minotti AM, Kountakis SE (1999) Management of abducens palsy in patients with petrositis. Ann Otol Rhinol Laryngol 108(9): 897-902.
11. Zhang N, Gevaert P, van Zele T, Perez-Novo C, Patou J, et al. (2005) An update on the impact of Staphylococcus aureus enterotoxins in chronic sinusitis with nasal polyposis. Rhinology 43(3): 162-168.

12. Homer JJ, Johnson IJ, Jones NS (1996) Middle ear infection and sixth nerve palsy. J Laryngol Otol 110(9): 872-874.

13. Allam AF, Schuknecht HF (1968) Pathology of petrositis. Laryngoscope 78(11): 1813-1832.

14. Yozu A, Suwa K, Mori M, Yamagata T, Mizuguchi M, et al. (2004) Magnetic resonance findings in a case of Gradenigo syndrome: widespread inflammation involving the paranasal sinuses and middle ear. No To Hattatsu 36(4): 334-338.

15. Hananya S and Horowitz Y (1997) Gradenigo syndrome and cavernous sinus thrombosis in fusobacterial acute otitis media. Harefuah 133(78): 284-286, 335.

16. Scardapane A, Del Torto M, Nozzi M, Elio C, Breda L, et al. (2010) Gradenigo's syndrome with lateral venous sinus thrombosis: successful conservative treatment. Eur J Pediatr 169(4): 437-440.

17. Goldstein NA, Casselbrant ML, Bluestone CD, Kurs-Lasky M (1998) Intratemporal complications of acute otitis media in infants and children. Otolaryngol Head Neck Surg 119(5): 444-454.

18. Thomas E R, Yvonne C A, Nicholas B S, Lesley M V (2011) Rare disease Conservative management of Gradenigo's syndrome in a child. BM] Case Reports. 2011; 2011. 
